\title{
The rise and fall of the metabolic syndrome
}

\author{
K. Borch-Johnsen • N. Wareham
}

Received: 14 December 2009/Accepted: 17 December 2009/Published online: 19 January 2010

(C) Springer-Verlag 2010

The clustering of risk factors for diabetes and cardiovascular disease (CVD) has been known for many decades. The origins of the notion of insulin resistance as a root cause of some forms of diabetes go back to 1936 when Himsworth [1] proposed the subdivision of diabetes into an insulinsensitive form and an insulin-resistant form, the latter typically associated with obesity. Even though a number of studies over the next decades identified additional CVD risk factors associated with diabetes and insulin resistance, it was only after Reaven's publication in 1988 [2] that the literature really started to proliferate. Coming from the field of physiology, Reaven elegantly proposed a hypothesis that could explain the link between a number of CVD risk factors including glucose, offering the suggestion that insulin resistance could be the common denominator underlying a syndromic clustering of metabolic risk factors. In other words, he offered a pathophysiological model that could be tested, confirmed or rejected. What followed was not the design of studies to test this underlying model, but rather a plethora of studies confirming the basic associations or proposing new markers that were also associated with insulin resistance. In either case, the observational evidence of association was all too often taken as evidence of causality. The literature proliferation popularised the concept of the 'metabolic syndrome', which, like a young

K. Borch-Johnsen $(\bowtie)$

Steno Diabetes Center,

Niels Steensensvej 2,

DK 2820 Gentofte, Denmark

e-mail: kbjo@steno.dk

N. Wareham

MRC Epidemiology Unit, Institute of Metabolic Science,

Addenbrooke's Hospital,

Cambridge, UK prince, grew rapidly from its baptism in 1988, confirmation by the WHO [3] in 1999 and ultimate coronation in heavily sponsored world conferences held in its own name. This ascent to the throne has not been without criticism [4], and recently the EASD and the American Diabetes Association (ADA) jointly declared in an extensive review of the topic [5] that: (1) the criteria are ambiguous and incomplete; (2) the rationale for thresholds is ill-defined; (3) the value of including diabetes in the definition is questionable; (4) the role of insulin resistance as the unifying aetiological factor is uncertain; (5) there is no clear basis for including or excluding other CVD risk factors; (6) the CVD risk value is variable and dependent on the specific risk factors present; (7) the CVD risk associated with the 'syndrome' appears to be no greater than the sum of its parts; (8) the treatment of the syndrome as a whole is no different from that of each of its components; and (9) the medical value of diagnosing the syndrome is unclear.

The 'metabolic syndrome' is clearly a cluster of risk factors, but is it truly a syndrome? Even though the definition of a syndrome varies between dictionaries, most would accept that a syndrome is more than just a cluster of events or risk factors. It could be defined as the sum of signs or symptoms that define a morbid state. In the case of the 'metabolic syndrome', the focus has been on identifying a single factor that could fulfil this role of an underlying morbid state, with insulin resistance [2] and central obesity [6] the prime contenders for this role. Unfortunately, as the WHO report published in this issue of Diabetologia [7] demonstrates, the role of each of these as the underlying mechanism for the clustering of risk factors remains unclear. This alone questions the existence of a 'metabolic syndrome'.

If we accept the term and agree that insulin resistance, central obesity, elevated blood pressure, elevated cholesterol/ LDL and triacylglycerol, reduced HDL and elevated glucose are components of the syndrome, then the next question is 
whether the syndrome is helpful to individuals, patients, health professionals or society. In practical terms, the issue is whether the concept of 'metabolic syndrome' helps: (1) identify individuals at risk of developing CVD or diabetes; (2) improve the care of patients with any of the components; or (3) further our understanding of why some people develop CVD or diabetes. The presence of the 'metabolic syndrome' does predict morbidity and mortality rates from CVD $[8,9]$, but that is not the relevant question. The real question is whether it predicts that risk over and above the prediction based on the individual components of the syndrome. The answer to this latter question has been consistently negative in the studies where it has been tested [10, 11]. Furthermore, there are numerous other risk prediction algorithms for CVD including the Framingham risk score [12] and the Heart Score [13], which outperform the 'metabolic syndrome' in predicting CVD events. Consequently, there is evidence that the syndrome has limited clinical use. Theoretically, the presence of the syndrome could change treatment modalities. Obviously, the presence of many risk factors should lead to a more multifactorial approach to treatment, but if there was a syndrome with a common underlying mechanism, then focusing therapy on this factor alone should be sufficient to treat the syndrome. The evidence is weak in relation to this, as we do not have specific treatments for central obesity and the available treatments that specifically target insulin resistance have not yet demonstrated a significant reduction in the risk of developing CVD [14]. Consequently, the presence of 'metabolic syndrome' does not inform the use of specific treatments, nor does it alter the treatment of individual risk factors. Clinicians regularly assess all components of the syndrome in the course of assessing cardiovascular risk, but no additional information is gained by condensing the results of such assessments into presence or absence of 'metabolic syndrome'. Finally, there is the question of whether use of the 'metabolic syndrome' has changed research. Judging from the number of publications identifiable in PubMed with 'metabolic syndrome' in the title or keywords, the concept has clearly attracted many researchers. But whether it has improved our understanding of why some people develop CVD or diabetes is much less clear. Indeed, lumping features of the syndrome together may well have obscured aetiological understanding, especially in a situation where an aetiological factor, e.g. fish oil intake, is associated in different directions with individual components of the syndrome.

In this issue of Diabetologia [7], a WHO Expert Consultation reports their view of the usefulness of the 'metabolic syndrome' as a concept and clinical tool. The WHO experts conclude that it has limited practical utility as a diagnostic or disease management tool and should not be ranked as a clinical diagnosis. In addition, they recommend that each country should develop cost-effective, context- specific diabetes and CVD risk identification and prevention strategies. Finally, they conclude that we need to return to science to understand the metabolic pathways underlying the development of diabetes and CVD. This report is a good reflection of the life-course journey of the 'metabolic syndrome, from its birth in 1988 and its rapid growth and confirmation by the WHO and International Diabetes Federation, through to the fading later years with critical reviews by the joint EASD/ADA group [5] and the present WHO Expert Consultation report [7]. In fact, it may be time to take the process even further, and to put the concept of the 'metabolic syndrome' to rest, either temporarily or permanently. We should also reflect on the possible harm done by focusing on the syndrome at the expense of diverting attention from simpler, but more precise risk prediction models, by attracting large research resources with limited outputs and by creating a questionable commercial alliance at meetings and world conferences, where the 'metabolic syndrome' became the magic bullet in publications and fundraising. Our best wishes for the 'metabolic syndrome' therefore should be: Rest in peace!

Duality of interest The authors declare that there is no duality of interest associated with this manuscript.

\section{References}

1. Himsworth H (1936) Diabetes mellitus: a differentiation into insulin-sensitive and insulin-insensitive types. Lancet i:127-130

2. Reaven GM (1988) Role of insulin resistance in human disease. Diabetes 37:1595-1607

3. World Health Organization (1999) Definition, diagnosis and classification of diabetes mellitus and its complications. Part 1: diagnosis and classification of diabetes mellitus. WHO, Geneva

4. Balkau B, Charles MA (1999) Comment on the provisional report from the WHO consultation. European Group for the Study of Insulin Resistance (EGIR). Diabet Med 16:442-443

5. Kahn R, Buse J, Ferrannini E, Stern M (2005) The metabolic syndrome: time for a critical appraisal. Diabetologia 48:1684-1699

6. Carr DB, Utzschneider KM, Hull RL et al (2004) Intra-abdominal fat is a major determinant of the National Cholesterol Education Program Adult Treatment Panel III criteria for the metabolic syndrome. Diabetes 53:2087-2094

7. Simmons RK, Alberti KGMM, Gale EAM et al (2010) The metabolic syndrome: useful concept or clinical tool? Report of a WHO Expert Consultation. Diabetologia doi:10.1007/s00125-009-1620-4

8. Ford ES (2005) Risks for all-cause mortality, cardiovascular disease, and diabetes associated with the metabolic syndrome: a summary of the evidence. Diabetes Care 28:1769-1778

9. Sattar N, McConnachie A, Shaper AG et al (2008) Can metabolic syndrome usefully predict cardiovascular disease and diabetes? Outcome data from two prospective studies. Lancet 371:1927-1935

10. Stern MP, Williams K, González-Villalpando C, Hunt KJ, Haffner SM (2004) Does the metabolic syndrome improve identification of individuals at risk of type 2 diabetes and/or cardiovascular disease. Diabetes Care 27:2676-2681 
11. Qiao Q, DECODE Study Group (2006) Comparison of different definitions of the metabolic syndrome in relation to cardiovascular mortality in European men and women. Diabetologia 49:28372846

12. Kohli P, Greenland P (2006) Role of the metabolic syndrome in risk assessment for coronary heart disease. JAMA 295:819821
13. Conroy RM, Pyörälä K, Fitzgerald AP et al (2003) Estimation of 10 -year risk of fatal cardiovascular disease in Europe: the SCORE project. Eur Heart J 24:987-1003

14. Lago RM, Singh PP, Nesto RW (2007) Congestive heart failure and cardiovascular death in patients with prediabetes and type 2 diabetes given thiazolidinediones: a meta-analysis of randomised clinical trials. Lancet 370:1129-1136 